\title{
A broadly-applicable unified closure relation for Taylor bubble rise velocity in pipes with stagnant liquid
}

\author{
E. Lizarraga-Garcia ${ }^{\mathrm{a}, *}$, J. Buongiorno $^{\mathrm{b}}$, E. Al-Safran ${ }^{\mathrm{c}}$, D. Lakehal $^{\mathrm{d}}$ \\ ${ }^{a}$ Department of Mechanical Engineering, MIT, Cambridge, MA 02139, USA \\ ${ }^{b}$ Department of Nuclear Science and Engineering, MIT, Cambridge, MA 02139, USA \\ ${ }^{c}$ Department of Petroleum Engineering, Kuwait University, Kuwait \\ ${ }^{d} A S C O M P$, Zurich, Switzerland
}

\begin{abstract}
Taylor bubble velocity in slug flow is a closure relation which significantly affects the prediction of liquid holdup (or void fraction) and pressure gradient in mechanistic models of slug flow for oil and gas pipe applications. In this work, we use a validated Computational Fluid Dynamics (CFD) approach to simulate the motion of Taylor bubbles in pipes; the interface is tracked with a Level-Set method implemented in a commercial code. A large numerical database is generated covering the most ample range of fluid properties and pipe inclination angles explored to date $\left(E o \in[10,700], M o \in\left[1 \cdot 10^{-6}, 5 \cdot 10^{3}\right]\right.$, and $\left.\theta \in\left[0^{\circ}, 90^{\circ}\right]\right)$. A unified Taylor bubble rise velocity correlation is extracted from that database. The new correlation predicts the numerical database with $8.6 \%$ absolute average relative error and a coefficient of determination $R^{2}=0.97$, and other available experimental data with $13.0 \%$ absolute average relative error and $R^{2}=0.84$ outperforming existing correlations and models.
\end{abstract}

Keywords: Taylor bubble, slug flow, terminal velocity, inclined pipes

\section{Introduction}

Two-phase slug flow is a common occurrence in wells, riser pipes and pipelines of crude oil and natural gas systems. Current predictive tools for two-phase flow

\footnotetext{
* Corresponding author

Email address: elizaga@it.edu (E. Lizarraga-Garcia)
}

Preprint submitted to International Journal of Multiphase Flow

October 14, 2016

C) 2016. This manuscript version is made available under the Elsevier user license http://www.elsevier.com/open-access/userlicense/1.0/ 
are based on either the mixture model or the mechanistic two-fluid model [1].

5 In the latter, slug flow is modeled as a sequence of fundamental units, also called slug units. Each unit contains a long bullet-shaped bubble, known as Taylor bubble, and a liquid portion with smaller homogeneously distributed bubbles, known as liquid slug. Thorough studies about the modeling of two-phase slug flow can be found in $[8,24,68$. The mechanistic model requires the use of closure relations to capture the transfer of mass, momentum and energy between the phases, in the respective conservation equations, so that integral flow parameters such as liquid holdup (or void fraction) and pressure gradient can be predicted. However, these closure relations typically carry the highest uncertainties in the model, since they are obtained empirically or through the use of overly simplified assumptions. In particular, significant discrepancies have been found between experimental data and closure relations for the Taylor bubble velocity in slug flow, which has been determined to strongly affect the pressure gradient and liquid holdup predicted by the mechanistic models of [2, 53, 57].

Taylor bubble velocity, $v_{T B}$, in slug flow is generally modeled based on the drift flux approach of [49],

$$
v_{T B}=C_{0} \cdot v_{m}+v_{d}
$$

where $v_{d}$ is the drift velocity of the bubble in a stagnant liquid, and $C_{0} \cdot v_{m}$ is 20 the contribution of the mixture velocity, $v_{m}$, which is the sum of the liquid and gas superficial velocities, $v_{m}=v_{S L}+v_{S g}$. The distribution parameter, $C_{0}$, is a dimensionless coefficient that depends on the velocity profile in the liquid, and is approximately the ratio of the maximum to the mean velocity. The present article is focused on the velocity and dynamics of the Taylor bubble in stagnant liquid, i.e., the second term of the RHS of equation 11. $v_{d}$.

Taylor bubble's dynamics are influenced by the viscous, inertial, gravitational, and interfacial forces acting on it. Assuming that the liquid transport properties are dominant $\left(\rho_{g} / \rho_{L} \ll 1, \mu_{g} / \mu_{L} \ll 1\right.$, where the subscripts $g$ and $L$ indicate the gas and liquid phases, respectively, $\rho$ is the density, and $\mu$ is the dynamic viscosity), dimensional analysis indicates the following four dimen- 


\begin{tabular}{l|l|l|l|l}
\multicolumn{5}{c}{ Table 1: Ranges of $M o$ for various oils 12] } \\
& $\rho_{L}\left[\mathrm{~kg} / \mathrm{m}^{3}\right]$ & $\sigma[\mathrm{N} / \mathrm{m}]$ & $\mu_{L}[\mathrm{~Pa} \cdot \mathrm{s}]$ & $M o$ \\
\hline \hline Heavy oil & 1100 & 0.03 & 2 & $5 \cdot 10^{3}$ \\
\hline High viscous oil & 1100 & 0.03 & 0.15 & $2 \cdot 10^{-1}$ \\
\hline Medium viscous oil & 1000 & 0.03 & 0.03 & $3 \cdot 10^{-4}$ \\
\hline Light oil & 900 & 0.03 & 0.007 & $1 \cdot 10^{-6}$ \\
\hline Very light oil & 800 & 0.03 & 0.001 & $5 \cdot 10^{-10}$ \\
\hline \hline
\end{tabular}

sionless pi-groups are sufficient to determine the bubble dynamics: the Froude number, $F r=v_{T B} / \sqrt{g d}$, where $g$ is the gravitational acceleration and $d$ is the pipe diameter, is the ratio of the bubble inertia to the gravitational forces; the Eötvös number, Eo $=\rho_{L} g d^{2} / \sigma, \sigma$ is the surface tension, is the ratio of the gravitational to interfacial forces; the Morton number, $M o=g \mu_{L}^{4} / \rho_{L} \sigma^{3}$, sometimes called the property number; and the inclination angle, $\theta$, measured from the horizontal. Note, however, that the choice of the pi-groups is not unique; for example, the inverse viscosity number, $N_{f}$, a combination of Eo and $M o$, can also be employed. Thus, Buckingham Pi-Theorem assures that the four pi-groups 40 are related by a unique function of the form $\operatorname{Fr}=f(E o, M o, \theta)$. In this article, a proposed correlation for this function is presented for an ample range of fluid properties and pipe inclination angles: $E o \in[10,700], M o \in\left[1 \cdot 10^{-6}, 5 \cdot 10^{3}\right]$, and $\theta \in\left[0^{\circ}, 90^{\circ}\right]$.

Table 1 shows the range of $M o$ for different types of oil. In this work, we focus on the upper range of the spectrum since ample work has been done using water, which has similar properties to very light oil, as will be described later. Furthermore, the oil and gas industry is currently moving towards the production of heavier oils, with the associated need to predict their behavior. 


\section{Literature review} a stagnant liquid is extensive. [20] and 23] studied analytically the limiting problem of negligible viscous force and surface tension where $F r$ is found to be constant. 75] performed a wide range of experiments and proposed a general graphical correlation of $F r$ as a function of $E o$ and $M o$, identifying regions experimentally the influence of liquid viscosity and surface tension on the bubble velocity, and proposed a velocity correlation for vertical tubes. 73 reported a correlation with three different regions based on $N_{f}$ for the vertical drift velocity and horizontal drift velocity, $v_{d}^{v}$ and $v_{d}^{h}$, respectively. [71] performed experiments $f\left(N_{f}, E o\right)$. They composed bi-power laws for two separate flow regions: large $N_{f}, N_{f}>200$, and small $N_{f}, N_{f}<10$, and connected them through a logistic dose response curve [55, 33]:

$$
\begin{aligned}
F r & =\frac{0.34}{\left(1+(14.793 / E o)^{3.06}\right)^{0.58}} /\left(1+\left(\frac{N_{f}}{31.08\left(1+(29.868 / E o)^{1.96}\right)^{0.49}}\right)^{a}\right)^{b}(2 a) \\
a & =-1.45\left(1+(24.867 / E o)^{9.93}\right)^{0.094} \\
b & =-1.0295 / a
\end{aligned}
$$

CFD numerical simulations have also been used to study slug flow mainly with 2D axisymmetric domain. [18, and 46] focused on the flow ahead and around the bubble. 4] analyzed a wide range of Taylor bubbles in vertical stagnant liquid columns using 2D axisymmetric simulations (Eo $\in[6,900]$, $\left.M o \in\left[4.72 \cdot 10^{-5}, 104\right], N_{f} \in[3.5,517]\right)$. [3] studied the hydrodynamics of pairs of consecutive Taylor bubbles in stagnant liquid. 37] studied experimentally and numerically the terminal velocities of clean and contaminated drops, as well as Taylor bubbles, in vertical pipes. In their terminal velocity correlation, they modified the coefficients of the correlations given by [29], who obtained the functional form of that empirical correlation through a force balance on the Taylor drop. The latter was deduced with a scaling analysis based on the field 
equations for two phases and the jump conditions at the interface. The range of applicability of their correlation is $E_{o} \in[4.8,228]$ and $M o \in\left[1 \cdot 10^{-12}, 1 \cdot 10^{4}\right]$. [60] performed simulations using VOF, including some limiting cases for inviscid liquid $\left(M o=0, N_{f}=\infty\right)$, and zero surface tension $(E o=\infty)$. Other 2D vertical axisymmetric studies were done by [13], 67] and [34].

The effect of the pipe inclination angle on the Taylor bubble dynamics has also been studied in the literature. Table 2 shows the Eo and $M o$ number ranges of these studies as well as the inclination angles covered, and tables 3 and 4 enumerate the correlations found in the literature for the Taylor bubble terminal velocity in inclined pipes. 77] described qualitatively the effect of tube inclination in closed tubes. [7] was the first to propose a correlation for inclined pipes. He claimed that $v_{d}^{h}$ can be different from zero in pipes with one end opened and partially filled with liquid and gas. In the open end, liquid drains out and gas enters the pipe, which moves the bubble forward inside due to the differences in liquid level at both sides of the bubble. These assumptions conflict with previous investigations of [49] and [22], who assumed $v_{d}^{h}=0$ in a closed horizontal pipe. 74, developed an ample experimental study covering $E o \in[4.9-490]$ and $M o \in\left[2.2 \cdot 10^{-11}, 1.5 \cdot 10^{4}\right]$. Furthermore, they proposed a correlation with a correction term $Q$ which is a function of $\Delta F r_{d}=F r_{d}^{v}-F r_{d}^{h}$. In their article, $F r_{d}^{v}$ and $F r_{h}^{v}$ are obtained by interpolation of the experimental data, and they give a graphical general dimensionless correlation for horizontal tubes. 28] performed an experimental study for $\theta \in\left[58^{\circ}, 90^{\circ}\right]$ and proposed a new correlation for bubble velocity in stagnant liquid. Equation $5 b$ is there justified for systems with large $N_{f}$ and Eo values (e.g., $N_{f}>300$ and $E o>100$ ) where $F r_{d}^{v}$ becomes constant. Furthermore, they assumed $v_{d}^{h}=0$. 63] and [1] studied experimentally and analytically the surface tension effects on the velocity of Taylor bubbles in inclined pipes, for which they used water and kerosene. [15] derived a semi-theoretical expression for the rise velocity of air bubbles in inclined pipes of stagnant water, and performed experiments with non-Newtonian fluids. [57] used the correlations of [73] for the horizontal drift velocity (equation 6a, and a modification of their vertical drift velocity. 62 
studied experimentally the motion of long bubbles in inclined tubes for $5^{\circ}$ to $90^{\circ}$ inclination angles, of Newtonian and non-Newtonian fluids. [26] investigated experimentally the effect of high oil viscosity on drift velocity for horizontal and inclined pipes using water and viscous oil. They proposed a new correlation for

perimental studies of slug flow in slightly deviated from horizontal tubes have been carried out by [10] and 64]. CFD simulations of Taylor bubbles in inclined pipes are computationally expensive, because axisymmetry cannot be assumed; as a result, there is no extensive study on the matter in the literature, and only 125 67] reported one inclined pipe case in their work.

As reviewed above, most multiphase models and correlations were developed with and for low viscosity fluids. For example, 7] and [28] used water, whose $M o=2.63 \cdot 10^{-11}$, a value which is within one order of magnitude of that for low viscosity oils. However, the oil and gas industry is currently moving towards constitute nearly $70 \%$ of the available reserves in the world, increasing the need to gain and improve the knowledge of the flow behavior of these fluids. When $\mu \in[0.150,1] \mathrm{Pa} \cdot \mathrm{s}$, oils are considered to be high viscous; medium viscosity oils when $\mu \in[0.030,0.150] \mathrm{Pa} \cdot \mathrm{s}$; and low viscosity oils when $\mu \in[0.001,0.030]$

$135 \mathrm{~Pa} \cdot \mathrm{s}$, corresponding to a $M o$ range of $\left[1 \cdot 10^{-10}, 5 \cdot 10^{3}\right]$. Furthermore, the range of $E o$ relevant to Taylor bubbles is $E o \in[10,700]$. Note that for $E_{o} \lesssim 4$ in 
Table 2: Experimental studies of Taylor bubbles in inclined pipes with a stagnant fluid

\begin{tabular}{|c|c|c|c|}
\hline & $\theta\left[{ }^{\circ}\right]$ & Eo & Mo \\
\hline 77 & $0-90$ & $3.48-4,000$ & $2.59 \cdot 10^{-11}-19.2$ \\
\hline 63 & $0-90$ & $50-10,000$ & $\sim 2.59 \cdot 10^{-11}$ \\
\hline 7] & $0-90$ & $50-340$ & $\sim 2.63 \cdot 10^{-11}$ \\
\hline 74 & $0-90$ & $4.9-490$ & $2.2 \cdot 10^{-11}-1.5 \cdot 10^{4}$ \\
\hline 28 & $58-90$ & 2,200 & $\sim 2.63 \cdot 10^{-11}$ \\
\hline 1$]$ & $0-90$ & 800 & $3.66 \cdot 10^{-6}$ \\
\hline 62 & $5-90$ & $21.9-629$ & $2.17 \cdot 10^{-11}-6.11 \cdot 10^{4}$ \\
\hline 26 & $5-90$ & $349 ; 776$ & $2.63 \cdot 10^{-11} ; 5.30 \cdot 10^{-1}-1.24 \cdot 10^{3}$ \\
\hline 31 & $0-90$ & $765-6,970$ & $0.258-49.2$ \\
\hline 48 & $0-90$ & 804 & $1.25 \cdot 10^{-3}-0.41$ \\
\hline Present study & $0-90$ & $10-700$ & $1 \cdot 10^{-6}-5 \cdot 10^{3}$ \\
\hline
\end{tabular}


Table 3: Terminal velocity correlations for Taylor bubbles in inclined pipes with a stagnant

fluid

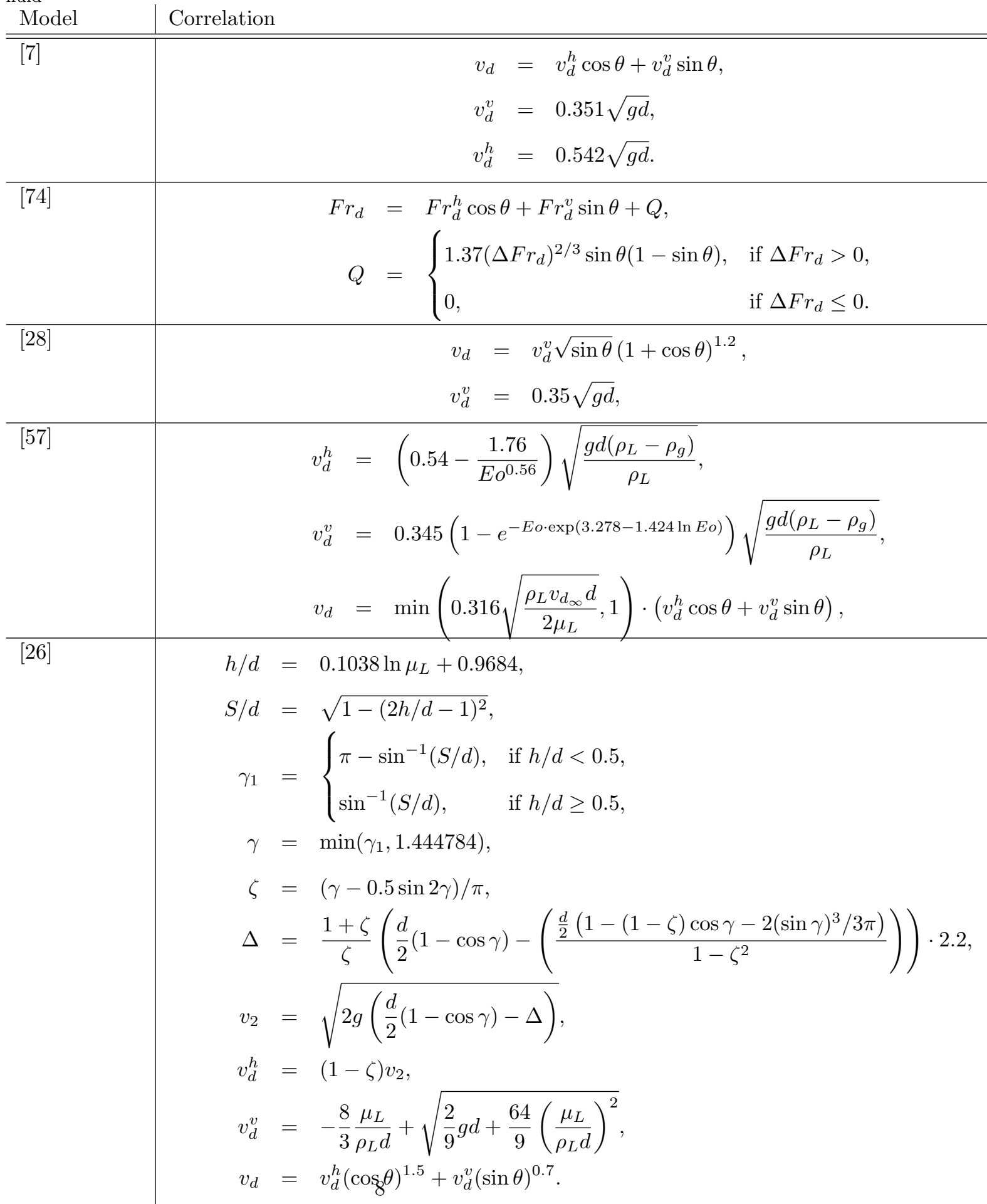


Table 4: Terminal velocity correlations for Taylor bubbles in inclined pipes with a stagnant fluid (continuation)

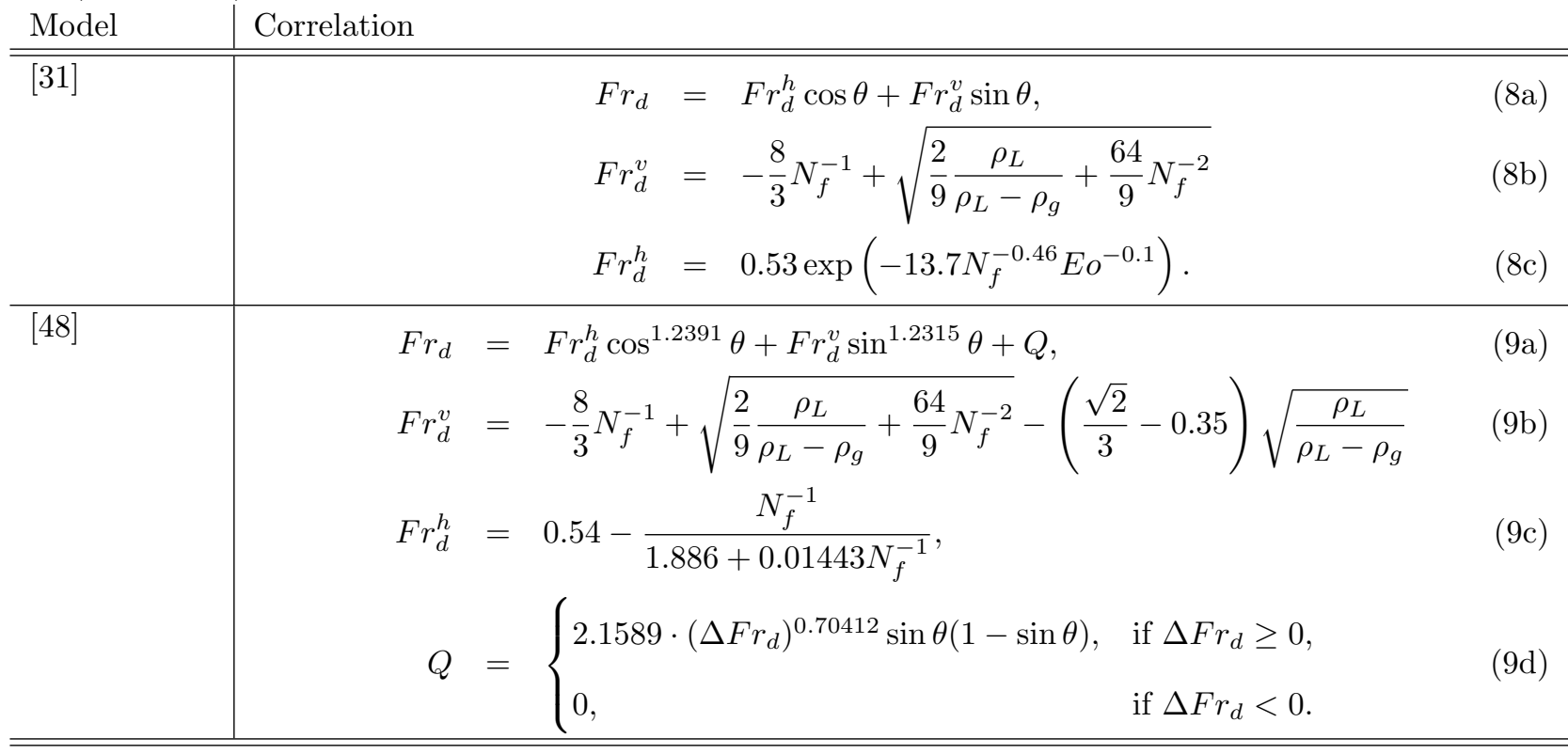

vertical pipes, the bubble occupies the entire pipe cross section and basically does not move [75]. On the other hand, 35] proposed that the maximum stable cap bubble size for vertical pipes occurs at approximately $E o \approx 900$, similar to what [36] predicted based on a two-dimensional Kelvin-Helmholtz instability analysis, and the experimental values reported by [19]. The implication is that the maximum pipe diameter for which Taylor bubbles and thus slug flow can exist corresponds to $E o \approx 900$. In such pipes, bubbly flow transitions to either cap flow or churn-turbulent flow as the gas velocity increases [16, 52, 58, 56]. It should be noted that this limit increases with increasing inclination angle, i.e. slug flow can exist in inclined pipes at larger diameters than in vertical pipes [30, 51]. However, the critical values of the Eo number at which this transition occurs are not available in the literature. 


\section{CFD simulations}

150

3.1. Mathematical model

3-D CFD Direct Numerical Simulations (DNS) are performed with the CMFD code 70, a finite-volume software developed at ASCOMP. The code uses structured meshes and MPI parallel-based algorithm to solve multi-fluid NavierStokes equations. Computer resources for this work include the supercomputers Ridge National Laboratory.

The code uses the one-fluid formulation approach, where the flow is described by one fluid with variable material properties, which vary according to a color function, which is advected by the flow. In the absence of phase change phenomena, the mass and momentum conservation equations are

$$
\begin{aligned}
\frac{\partial \rho}{\partial t}+\nabla \cdot(\rho \mathbf{v}) & =0 \\
\frac{\partial(\rho \mathbf{v})}{\partial t}+\nabla \cdot(\rho \mathbf{v} \mathbf{v}) & =-\nabla p+\nabla \cdot \overline{\bar{\sigma}}+\rho \mathbf{g}+\mathbf{F}_{s}
\end{aligned}
$$

where $t$ is the time, $\mathbf{v}$ is the velocity vector, $p$ is the pressure, $\overline{\bar{\sigma}}=\mu\left(\nabla \mathbf{v}+\nabla^{T} \mathbf{v}\right)$ is the viscous stress tensor, $\mathbf{g}$ is the gravity vector, and $\mathbf{F}_{s}=\sigma \kappa \mathbf{n} \delta(\phi)$ is the surface tension term where $\kappa=\nabla \cdot(\nabla \phi /|\nabla \phi|)$ is the surface curvature, $\mathbf{n}$ is the vector normal to the interface, and $\delta$ is a smoothed delta function centered at the interface. In this work, the color function used is based on the Level Set (LS) method [54, 66], where the interface is represented by a continuous and monotonous function $\phi$ that represents the distance to the interface at which $\phi=0$. The LS advection equation is given by

$$
\frac{\partial \phi}{\partial t}+\mathbf{v} \cdot \nabla \phi=0
$$

and material properties such as density and viscosity are updated locally based on $\phi$, and smoothed across the interface using a smooth Heaviside function. Furthermore, the pipe is modeled as an embedded surface and represented in the fluid by the so-called Solid Level Set function where $\phi_{s}=0$ is the fluid-solid 

mesh is locally refined next to the pipe walls.

Simulations are carried out using the 2nd-order Hybrid Linear/Parabolic Approximation (HLPA) scheme [76] for the discretization of the convective fluxes. An implicit 1st-order scheme is used for the time marching, where the time-step

170 is adaptive and bounded by a Courant number fixed between 0.5 and 0.9 to guarantee stability of the simulations. The SIMPLEC (Semi-Implicit Method for Pressure Linked Equations-Consistent) algorithm is used for the pressurevelocity coupling [21]. For the level-set, a 3rd-order WENO scheme is used for re-distancing, and mass conservation is enforced using global and local massconservation schemes 39. Finally, the solvers used depend on the simulation. For high viscosity cases where the Navier-Stokes equations tend to elliptic, the R-cycle adaptive Algebraic Multigrid (AMG) method is used [59]. Otherwise, either the incomplete lower-upper decomposition method Strongly Implicit Procedure (SIP) 65] or the Generalized Minimum Residual method (GMRES) 61] are used, the latter with hypre AMG preconditioning [25] of the parallel PETSc (Portable, Extensible Toolkit for Scientific Computation) library [5].

\section{2. $C F D$ simulations}

The text matrix explored in this study covers the ranges $M o \in\left[1 \cdot 10^{-6}, 5 \cdot 10^{3}\right]$ and $E o \in[10,700]$, and consists of the 25 logarithmically spaced cases shown in figure 1 and summarized in table 2. It can be observed that there are more points for lower Eo as there is a sharp transition in the velocity behavior for $E_{O} \approx 20$, as shown later. For each case, up to seven inclination angles $\left(5^{\circ}, 15^{\circ}, 30^{\circ}, 45^{\circ}, 60^{\circ}, 75^{\circ}, 90^{\circ}\right)$ are simulated for a total of 147 . This test matrix constitutes a substantial expansion of the ranges of parameters explored in previous studies.

The simulations start with a single bubble in still liquid and finish when the bubble reaches its terminal velocity. The initial bubble shape is a cylinder closed at the ends by two semi-spherical caps, and is placed inside a closed pipe embedded in the numerical domain. It should be emphasized that the 


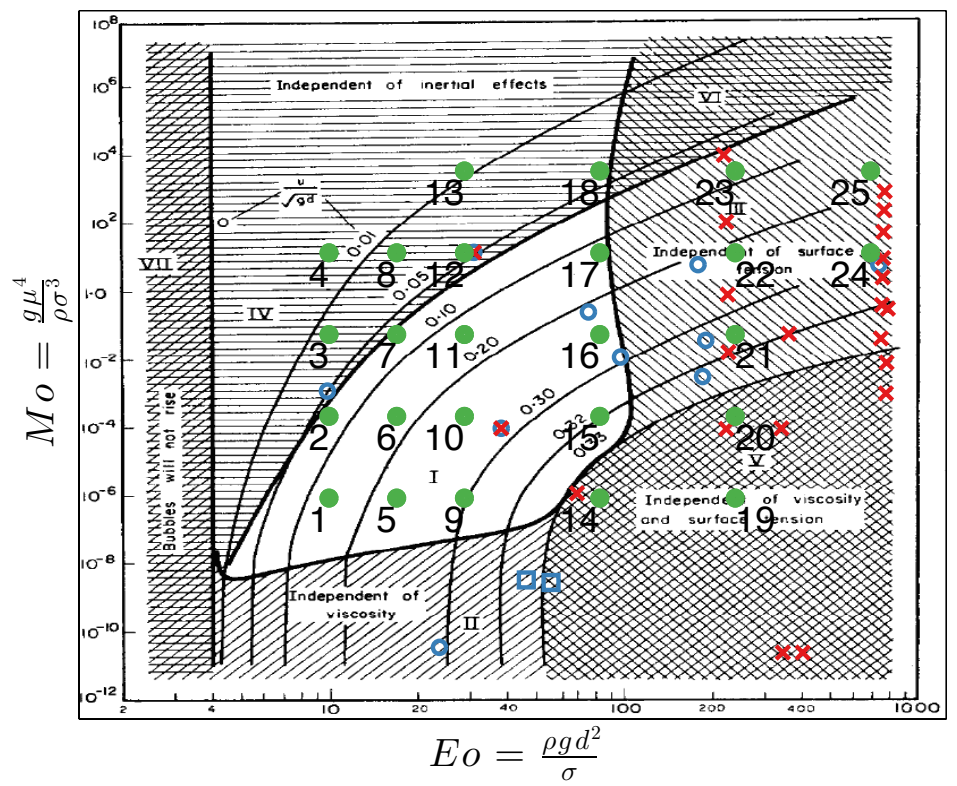

Figure 1: The map of 75] with the location of the numbered numerical database generated to obtain the $F r$ correlation of equations $16(\bullet)$, numerical cases used to validate the CMFD code ( $\circ$, where $\square$ are the experiments performed in this study), and experimental cases gathered from the literature $77,74,62,26,48$ to test the proposed correlation $(\times)$. 

as we extract the data after the bubble has reached its terminal velocity and shape. We have verified it through direct simulation of a few cases for evaluation of these sensitivities. Bubble volume ranges from $\lambda=1$ to 1.35 , where the parameter $\lambda$ is the ratio of the sphere-volume equivalent bubble diameter to the pipe diameter, $\lambda=d_{e} / d$. The pipe is the reference frame, thus its velocity is set to zero, and its length is case dependent so that boundary effects do not affect the results, with typical values around $10 d$. The boundary conditions of the numerical domain are symmetry planes where the normal velocity and pressure gradient components are set to zero. The no-slip condition at the wall is imposed through a relaxation term which acts as a distributed momentum sink reducing the fluid velocity as the indicator function goes to zero [6]. The structured mesh size is dependent on the case study and is refined until the terminal velocity converges, capturing the Kolmogorov scale, $\eta$, estimated as $\eta \sim d \cdot R e^{-3 / 4}$, where $R e=F r \cdot N_{f}$. Re ranges between 0.01 cells ranges from 1 million up to 8.5 million cells, with mesh refinement at the pipe wall and a maximum cell aspect ratio of 3.
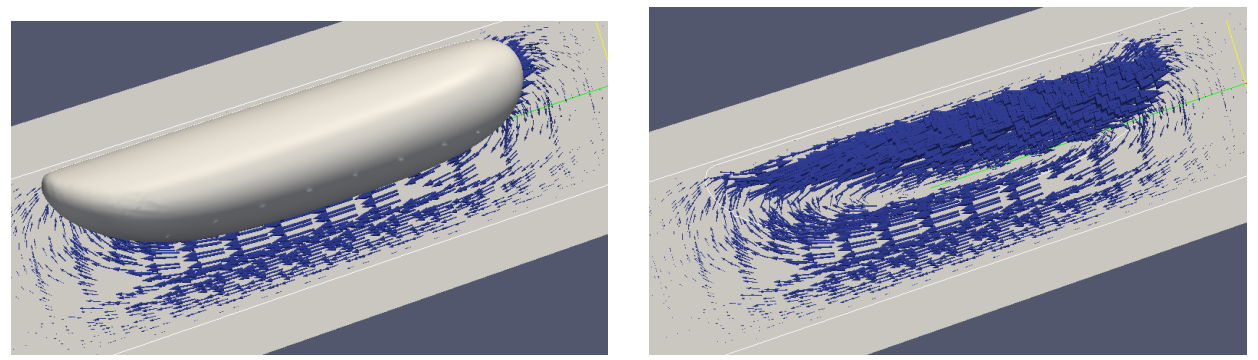

Figure 2: Snapshot of the Taylor bubble and the streamlines for $E o=29, M o=0.07$, and $\theta=30^{\circ}$.

Furthermore, two results are analyzed based on two verified assumptions: the Taylor bubble length does not affect its rising velocity in inclined pipes and there exists a lubricating film between the Taylor bubble and the pipe wall at all inclinations. The first assumption is justified in AppendixC. The 
second assumption is verified by application of the film drainage and breakup criterion proposed in [43. This criterion states that film breakup does not occur if the non-dimensional bubble passage time, $\bar{t}_{\text {bubble }}=t_{\text {bubble }} / \tau<0.01$, where $\tau$ is the characteristic drainage time. The bubble passage time can be calculated as $t_{\text {bubble }}=L / v_{T B}$, where $L$ is the bubble length, and $v_{T B}$ is the bubble velocity. The characteristic time is $\tau=3 \mu_{L} R /\left(2 \rho g \cos \theta h_{c}^{2}\right)$, where $h_{c}$ is the critical thickness. The critical thickness order-of-magnitude estimate for the cases studied in steel pipe is $h_{c} \sim 1 \mu \mathrm{m}$, using the expressions of [72]. The most critical case we simulate is that for $\theta=5^{\circ}$. At that point, the critical length below which the film does not break is calculated based on the aforementioned criterion as

$$
(L / d)_{c}=0.01 v_{T B} \tau / d .
$$

The results from equation 12 are reported in the last column of table A.7 of AppendixA The lowest value of $(L / d)_{c}$ is three hundred, that is, the length of the Taylor bubble simulated is much lower than the critical length calculated. Thus, the lubricating film is indeed present above the Taylor bubble in all cases simulated.

Representative snapshots of the shape and streamlines of a Taylor bubble with $E o=29, M o=0.07$, and $\theta=30^{\circ}$ are shown in figure 2. We validated the code using data from the literature for the Taylor bubble terminal velocity in vertical and inclined pipes. The calculated bubble shape was compared with experimental data from [50] for a case with $E o=192$ and $M o=4.75 \cdot 10^{-2}$, and the calculated velocity vectors were compared to experimental Particle Image Velocimetry (PIV) data from 14 for a case with $E o=98.4$ and $M o=1.52$. $10^{-2}$. Details are reported in AppendixB and more can be found in [40, 42, 41].

\section{Results}

For vertical pipes, the $F r$ obtained numerically is compared with the results obtained using the correlations of [71] and [37]. The average terminal error of the simulations is $3.9 \%$ and $7.4 \%$, with a standard deviation of $1.1 \%$ and 
71] appears to be an accurate tool to predictcalculate the terminal velocity of Taylor bubbles in vertical pipes, although the correlation of [37] predicts more accurately for air-water systems with $E_{O}<30$. Furthermore, the dimensionless film thickness, $\bar{h}=h / d$, is compared with the correlation of [44. Although the authors developed the correlation using experimental data where interfacial tension can be neglected, they proposed a generalization of the model to the cases where interfacial tension cannot be neglected. According to our data, this works well: the average error is $3.1 \% \pm 1.3 \%$.

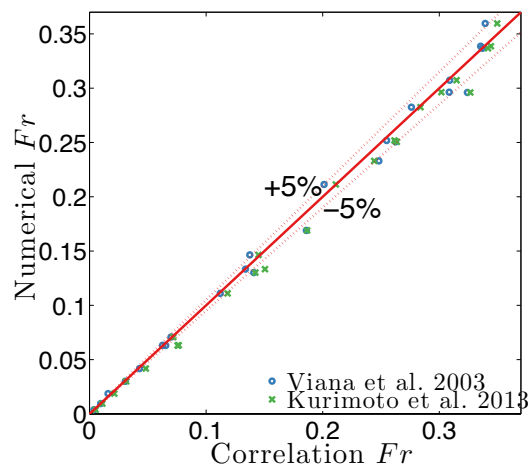

Figure 3: Taylor bubble terminal Fr numerical results compared with the values obtained from literature correlations for vertical pipes

As far as inclined pipes are concerned, figure 4 shows the results from a few representative simulations of ours and the predictions of the correlations of [7, [28, [57], 26], 31, and [48. (see tables 3 and 47. Note the agreement of the correlations with each other and the numerical data is rather poor, which further motivates the need for a new broadly-applicable and accurate correlation. The maxima results from the competing effects of drag coefficient (lower at lower angles, where most of the liquid bypasses the bubble through a larger flow area), and buoyancy (higher at higher angles). Figure 5 shows this behavior for case $16\left(E o=83.7\right.$ and $\left.M o=7.07 \cdot 10^{-2}\right)$ and three inclination angles: at $\theta=90^{\circ}$, the bubble is axisymmetric and the drag coefficient is maximum. As 

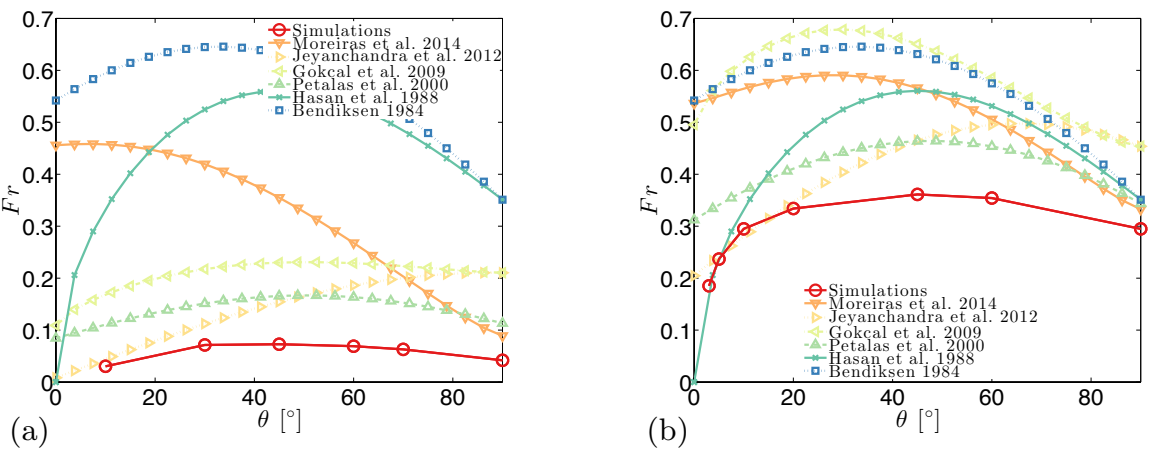

Figure 4: $F r$ as a function of $\theta$ for $E o=31.2$ and $M o=19.2$ (a) and $E o=38.6$ and $M o=1.17 \cdot 10^{-4}$ (b) compared with experimental correlations from the literature

the pipe inclines, the bubble front area is reduced as it approaches the pipe wall, and axisymmetry no longer applies. It should be noted that the longitudinal and radial directions, $z$ and $r$, respectively, are normalized by the pipe radius, $R$, so that the dimensionless longitudinal and radial directions, $z^{*}$ and $r^{*}$, respectively, are used.

Our complete numerical database is shown in figure 6. The plots are superimposed over the Eo - Mo map, and then within each plot the dependence of $F r / F r_{v}$ on the inclination angle is shown, where $F r_{v}$ is the vertical pipe $F r$. The continuous lines in the plots are from the models explained in the next section. For each case, the maximum value occurs at around $\theta=45^{\circ}$. Furthermore, two distinct regions are observed regarding the maximum value of the normalized $F r,\left(F r / F r_{v}\right)$ : for $E o>20$, the amplitude of $F r / F r_{v}$ is small for low $M o$ and high Eo, and slightly higher for high $M o-$ low Eo cases. However, for $E o<20$, this amplitude is significantly bigger. At this Eo range and vertical pipes, the bubble presents a bulge of higher radius in its bottom, making the liquid film effectively thinner and increasing the form drag. For example, the bulge can only be observed for the cases with $E o=10$ and 17 of figure 7. where the bubble bottom shape is shown for the cases with $\theta=90^{\circ}$, and $M o=7.1 \cdot 10^{-2}$ and 19 , respectively. As the pipe inclines, the bubble moves to the wall and the flow area increases in higher proportion than for the cases with 

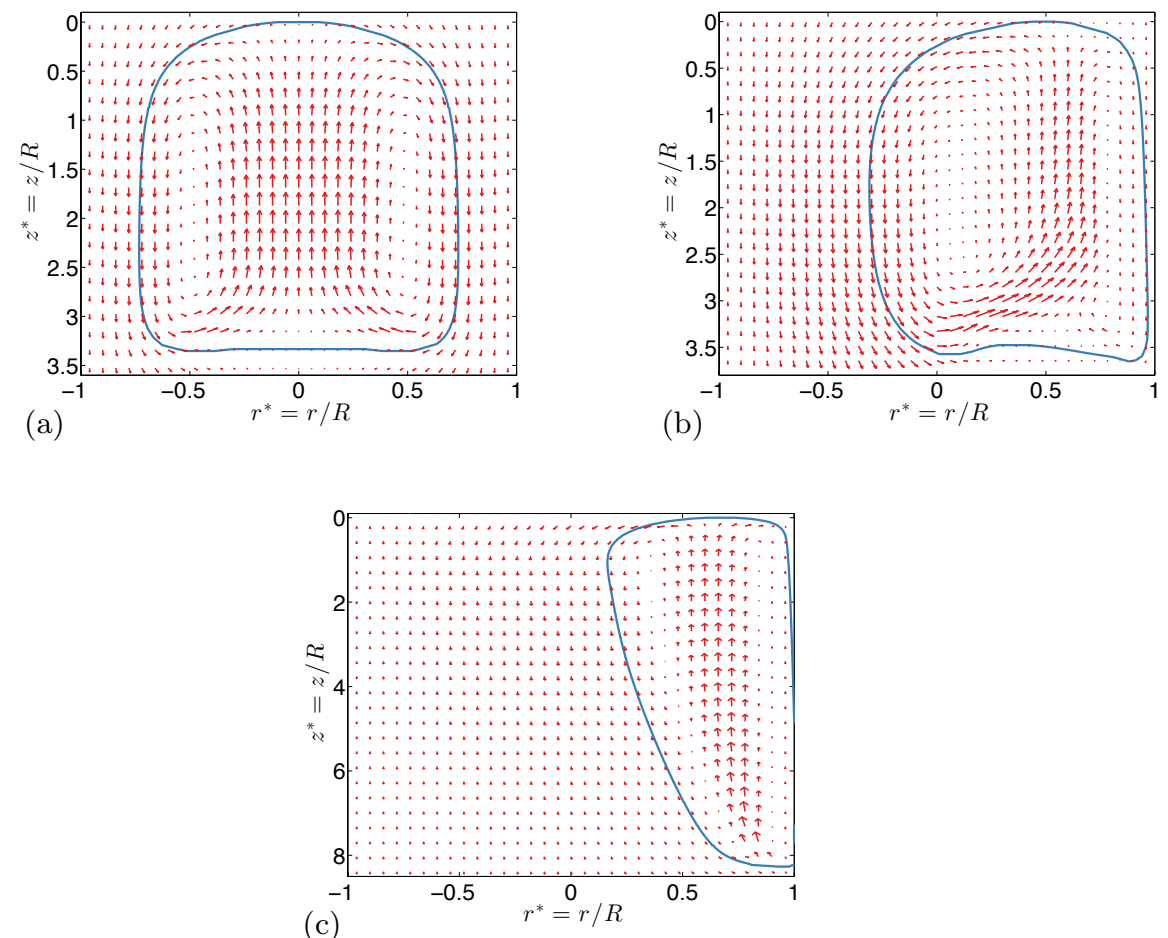

Figure 5: Bubble shape and velocity vectors at a cross sectional cut for case 16 (Eo $=83.7$ and $M o=7.07 \cdot 10^{-2}$ ), at $\theta=90^{\circ}(\mathrm{a}), \theta=45^{\circ}(\mathrm{b})$, and $\theta=5^{\circ}$ (c) 
$E o>20$, as the disappearance of the bulge also contributes to this increment.

Another feature observed in the map is the flat profile for low Mo and high Eo cases, where the velocity drops rapidly for low inclination angles.

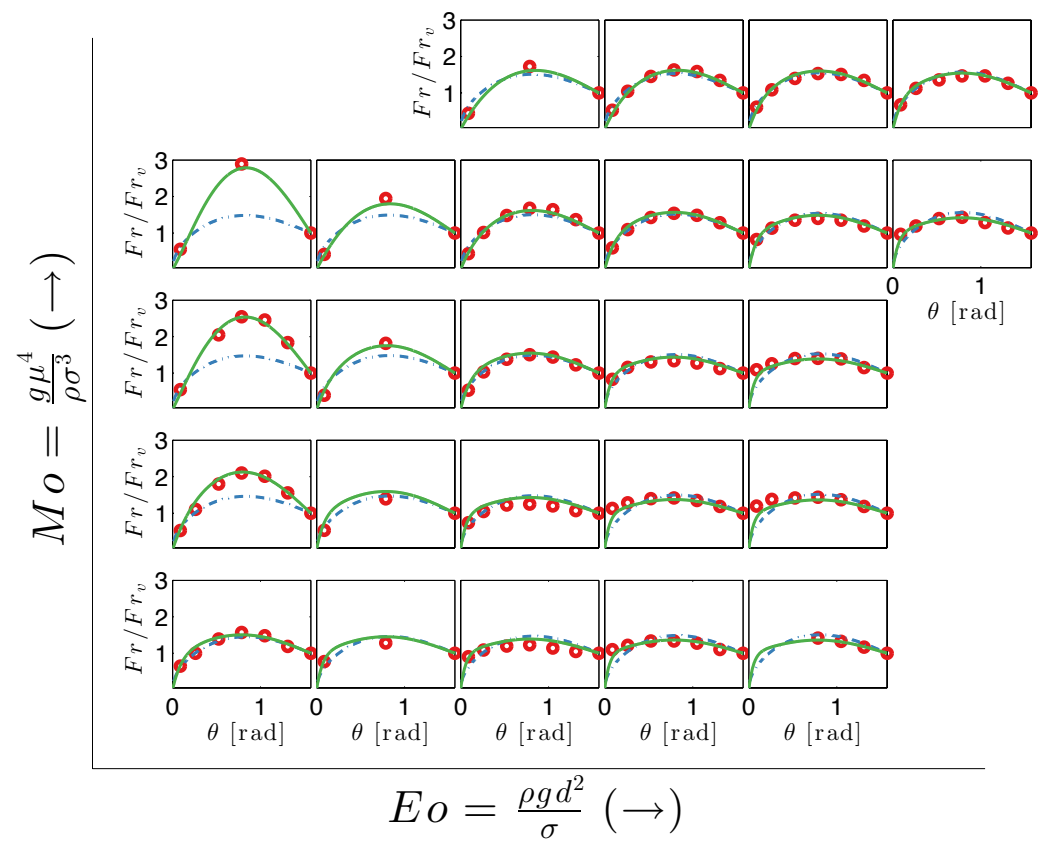

Figure 6: Normalized Taylor bubble terminal $F r\left(F r / F r_{v}\right)$, for the values of Eo-Mo shown in figure 1 numerical results (०), correlation of equation $14(---)$, and correlation of equations $16(-)$.

\section{Proposed new correlation for Taylor bubble velocity}

We will now use the numerical database to generate a correlation that can serve as closure relation for Taylor bubble velocity in mechanistic models of slug flow. Following the procedure of [28, we can start by considering the balance of buoyancy and drag force that determines the terminal velocity of a Taylor bubble; after the pi-groups are introduced, the force balance yields an expression 

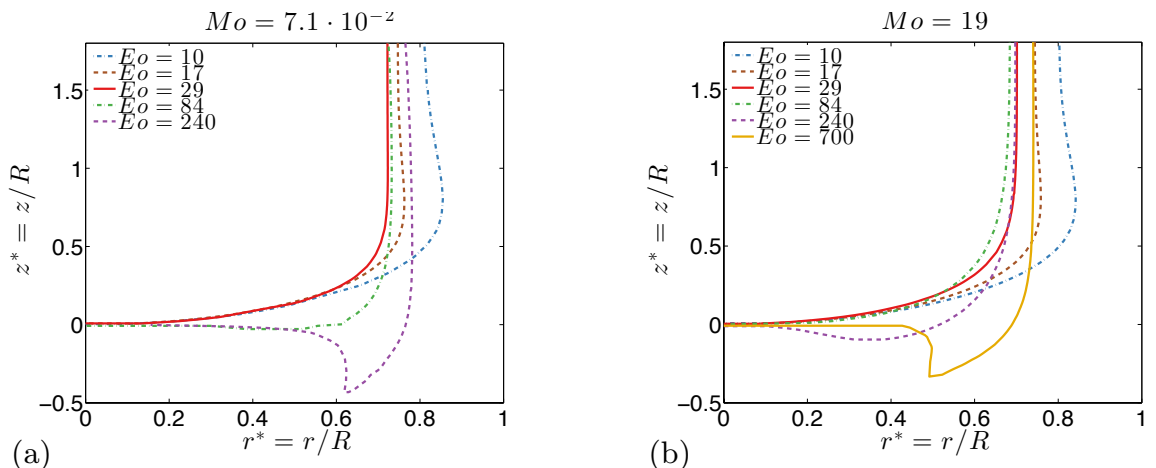

Figure 7: Taylor bubble bottom shape for the cases with $\theta=90^{\circ}$, and $M o=7.1 \cdot 10^{-2}$ (a) and 19 (b). The bulge at the bottom decreases with Eo and disappears for Eo $>20$.

for Fr as follows:

$$
F r=F r_{v}(\sin \theta)^{1 / 2} g(E o, M o, \theta),
$$

where the function $g(E o, M o, \theta)$ is assumed to be $(1+\cos \theta)^{E o^{a} M o^{b}}$ so that

$$
F r=F r_{v}(\sin \theta)^{1 / 2}(1+\cos \theta)^{E o^{a} M o^{b}},
$$

and the exponents $a$ and $b$ are then fitted to maximize the accuracy of equation 14 in reproducing the numerical database. The fit is performed using the nonlinear optimization algorithms built in 47. However, the results are not satisfactory at low inclination angles for high $M o$ and low Eo values (see figure 6). Thus, a new function is proposed:

$$
F r=F r_{v}(1-\exp (-b(E o, M o) \theta))(1+c(E o, M o) \sin (2 \theta)) \text {, }
$$

where the exponential function reproduces the rapid velocity decay observed at low inclination angles, and the sinusoidal function reproduces the maximum velocity at $\theta=45^{\circ}$. The coefficients $b(E o, M o)$ and $c(E o, M o)$ are chosen to yield the most accurate fit:

$$
\begin{aligned}
F r & =F r_{v}(1-\exp (-b(E o, M o) \theta))(1+c(E o, M o) \sin (2 \theta)) \\
b(E o, M o) & =47.06 F r_{v}+4 \\
c(E o, M o) & =-0.9118 F r_{v}+0.67+\frac{-0.0148\left(\log _{10} M o\right)^{2}+0.125 \log _{10} M o+0.9118 F r_{v}+1.118}{\left(1+(E o / 20)^{8}\right)^{8}}
\end{aligned}
$$


The coefficient $c(E o, M o)$ is predicted with a logistic dose-response curve that connects the two differentiated regions described above [55, 33. In order to use this this new correlation for any values of $E o, M o$ (or $N_{f}$ ) and $\theta$, first calculate $F r_{v}$ from the correlation of [71, equations 2. Then, calculate the coefficients $b$ and $c$ from equations $16 \mathrm{~b}$ and $16 \mathrm{c}$, respectively, and finally, calculate $\mathrm{Fr}$ from equation 16a. Note that equation 16 is a continuous and smooth function of all the relevant variables and thus suitable for use as a closure relation in codes that implement the mechanistic model.

\section{Performance of the new correlation}

In this section, the performance of the proposed correlation (equations 16) is compared with the performance of the other correlations and models in the literature: [7, 28], [57, 26], 31], and [48] (see tables 3 and 44). Two databases are used for the comparison: the numerical database (section 6.1) and the experimental database (section 6.2). In the comparison we shall use several statistical parameters that characterize the performance of the models and are explained in AppendixD.

\subsection{Numerical database}

Table 5 summarizes the error statistics. Figure 8 a reports the values of $\mathrm{Fr}$ from the numerical simulations versus the predictions of equations 16 . On its right, figure $8 \mathrm{~b}$ shows the cumulative plot of the relative error, $E_{2}$ (equation D.2), for the numerical database. It can be seen that the proposed correlation outperforms the other ones: using the proposed correlation, up to $70 \%$ of the cases show a relative error, $E_{2}$, of less than $10 \%$. On the other hand, the other literature models predict less than $10 \%$ of the cases with a relative error, $E_{2}$, of less than $10 \%$.

Of course the excellent performance of our correlation in reproducing the numerical database is not surprising since it was developed as a best fit of the numerical database, and the functions proposed are able to capture the velocity trends. 


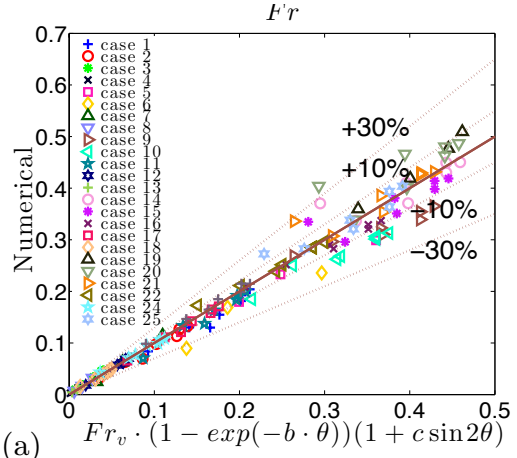

(a) (b)

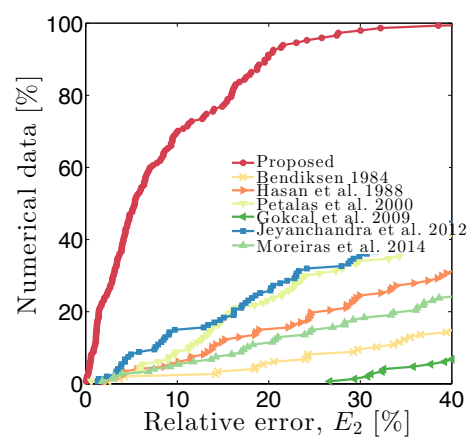

Figure 8: $F r$ numerical values versus the predictions of the proposed correlation, equations 16 (a), and cumulative plot of the relative error, $E_{2}$, for the numerical database using the proposed and literature correlations (b).

Table 5: Statistical parameters of the models analyzed using the numerical database generated

\begin{tabular}{|c|c|c|c|c|c|c|c|}
\hline Model & $E_{1}$ & $E_{2}$ & $E_{3}$ & $E_{4}$ & $E_{5}$ & $E_{6}$ & $\mathrm{R}^{2}$ \\
\hline [7] & 860 & 860 & 2400 & 0.34 & 0.34 & 0.16 & -5.79 \\
\hline 48 & 430 & 450 & 1400 & 0.22 & 0.22 & 0.14 & -2.22 \\
\hline 28 & 530 & 540 & 1300 & 0.22 & 0.23 & 0.16 & -2.55 \\
\hline 31 & 120 & 130 & 250 & 0.10 & 0.11 & 0.092 & 0.086 \\
\hline 57 & 120 & 120 & 190 & 0.096 & 0.097 & 0.060 & 0.40 \\
\hline 26 & 220 & 220 & 310 & 0.21 & 0.21 & 0.10 & -1.63 \\
\hline Proposed (equations 16 & 1.4 & 8.6 & 12.1 & 0.0041 & 0.017 & 0.026 & 0.976 \\
\hline
\end{tabular}




\subsection{Experimental database}

We have also tested the models against the experimental data shown in figure 9a, and reported in AppendixA. It is worth mentioning that these experimental data were not used in fitting the coefficients of equations 16 . The 178 experimental datapoints, gathered from the literature [77, 74, 62, 26, 48, cover the whole range of inclination angles and an ample region in the Eo-Mo map. Figure $9 \mathrm{~b}$ depicts the results of $F r / F r_{v}$ for both the experimental values and the predicted ones with equations 16 . In general, the model predicts well the behavior except for cases 6 and 7 , which were performed with an air/water mixture with $M o=2.63 \cdot 10^{-11}$ outside the ranges of our numerical database, as seen in figure 9 a.

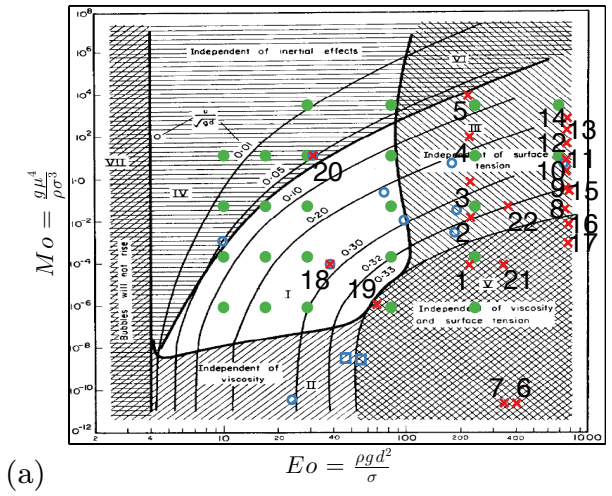

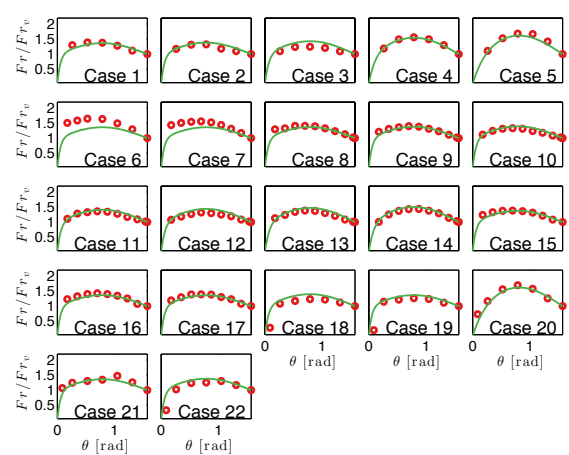

(b)

Figure 9: Numbered experimental cases gathered from the literature [77, 74, 62, 26, 48, to test our model $(\times)$ located in the map of 75$]$ together with the numerical database developed in this study $(\bullet)$ and the cases to validate the CMFD code $(\circ, \square)(a)$, and the Taylor bubble terminal $F r$ over the vertical $F r_{v}, F r / F r_{v}$, for the experimental database: experimental results (o), and predictions of equations $16(-)(\mathrm{b})$.

Figure 10 depicts the experimental values of $F r$ versus the proposed correlation. Furthermore, the cumulative error plot of the relative error, $E_{2}$, for the experimental database is shown in figure $10 \mathrm{p}$. It can be seen that the proposed correlation again outperforms the other ones: using the proposed model, up to $70 \%$ of the cases show a relative error, $E_{2}$, of less than $10 \%$. On the other hand, 
around $20 \%$ of the cases show a relative error, $E_{2}$, of less than $10 \%$ using the literature models. The three datapoints of figure 10 a that are well below the $30 \%$ prediction error correspond to $\theta=5^{\circ}$ of [62] (cases 18, 19 and 22 of figure 9b) where the velocity is remarkably low.
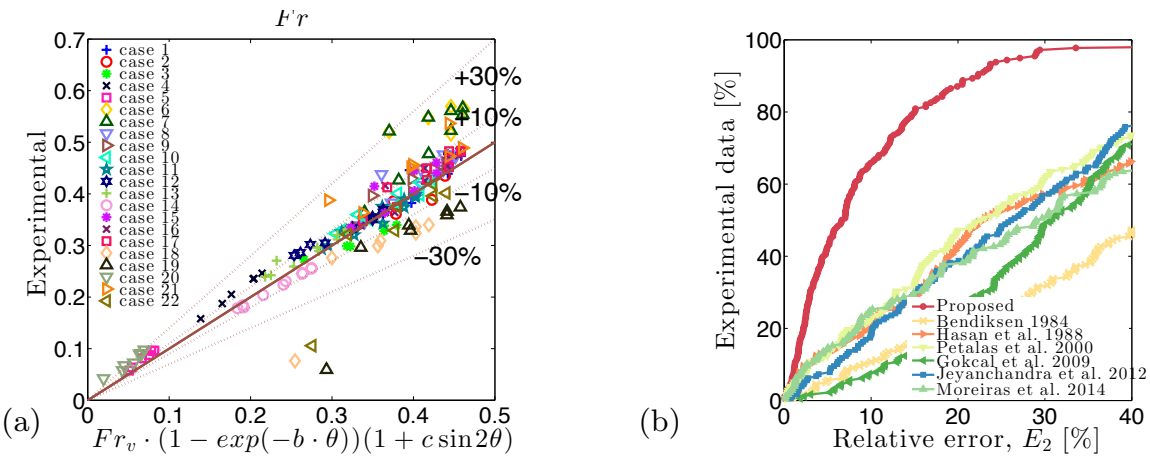

Figure 10: $F r$ experimental values versus the predictions of the proposed correlation, equations 16 (a), and cumulative plot of the relative error, $E_{2}$, for the experimental database using the proposed and literature correlations (b).

325

Finally, table 6 shows the statistical parameters that characterize the performance of the models. It is worth noting that our proposed correlation reports a coefficient of determination $R^{2}=0.84$, while without the cases 6 and 7 $R^{2}=0.87$. The second best model is that of [31] with $R^{2}=0.17$. Moreover, both $E_{2}$ and $E_{3}$ are $13 \%$ and $39 \%$ using our proposed model, respectively, while they are $37 \%$ and $56 \%$, respectively, using the model of [31.

\section{Conclusions}

In this work, a new unified correlation for the terminal velocity of Taylor bubbles in pipes with a stagnant liquid for an ample range of fluid properties and pipe inclination angles was presented (equations 16). The correlation was developed based on a CFD-generated database covering Eo $\in[10,700], M o \in[1$. $\left.10^{-6}, 5 \cdot 10^{3}\right], \theta \in\left[0^{\circ}, 90^{\circ}\right]$, and within those ranges it clearly outperforms current correlations when tested against both the numerical database and experimental data: the absolute average relative error is $8.6 \%$ and $13 \%$, while the coefficient 
Table 6: Statistical parameters of the models analyzed using the experimental database shown in figure $9 a$

\begin{tabular}{l|l|l|l|l|l|l|l} 
Model & $E_{1}$ & $E_{2}$ & $E_{3}$ & $E_{4}$ & $E_{5}$ & $E_{6}$ & $\mathrm{R}^{2}$ \\
\hline \hline$[7]$ & 110 & 110 & 190 & 0.20 & 0.20 & 0.13 & -2.90 \\
\hline$[48]$ & 63 & 64 & 130 & 0.12 & 0.13 & 0.10 & -0.81 \\
\hline$[28]$ & 66 & 69 & 130 & 0.11 & 0.12 & 0.12 & -0.79 \\
\hline$[31]$ & 34 & 37 & 56 & 0.087 & 0.095 & 0.068 & 0.17 \\
\hline$[57]$ & 35 & 39 & 65 & 0.080 & 0.093 & 0.087 & 0.052 \\
\hline$[26]$ & 51 & 52 & 94 & 0.12 & 0.13 & 0.083 & -0.50 \\
\hline Proposed (equations 16 & 0.32 & 13 & 39 & -0.104 & 0.032 & 0.048 & 0.84 \\
\hline \hline
\end{tabular}

of determination $R^{2}$ coefficient is 0.97 and 0.84 , respectively. The second best correlation reports an absolute average relative error of $120 \%$ and $37 \%$, and a coefficient of determination $R^{2}$ equal to 0.40 and 0.17 , respectively. The new correlation is recommended for use as a slug flow closure relation in codes that implement the mechanistic model.

\section{Acknowledgments}

This work was sponsored by the MIT-Kuwait Center for Natural Resources and the Environment. Enrique Lizarraga-Garcia is also thankful to the "Caja Madrid" Foundation for his postgraduate fellowship. The authors would like to express their gratitude to Dr. Matteo Bucci from MIT for his assistance with the experiments, and to Dr. Chidu Narayanan and Mr. Daniel Caviezel from ASCOMP for their support with the code. This research used the computational resources of the Oak Ridge Leadership Computing Facility at the Oak Ridge National Laboratory, which is supported by the Office of Science of the U.S. Department of Energy under Contract No. DE-AC05-00OR22725. 
Table A.7: Numerical database for the vertical pipe simulations

\begin{tabular}{|c|c|c|c|c|c|c|c|}
\hline \multirow[t]{2}{*}{ Case } & \multirow[t]{2}{*}{ Eo } & \multirow[t]{2}{*}{$M o$} & \multirow[t]{2}{*}{$N_{f}$} & \multicolumn{2}{|l|}{$F r$} & \multirow[t]{2}{*}{$R e$} & \multirow[t]{2}{*}{$(L / d)_{c}$} \\
\hline & & & & Simulated & 71 & & \\
\hline$\overline{c 1}$ & 10 & $1 \cdot 1 \cdot 10^{-6}$ & 180 & 0.13 & 0.14 & 23 & $1.4 \cdot 10^{3}$ \\
\hline 2 & 10 & $2.7 \cdot 10^{-4}$ & 44 & 0.063 & 0.066 & 2.8 & $2.3 \cdot 10^{3}$ \\
\hline 3 & 10 & $7.1 \cdot 10^{-2}$ & 11 & 0.019 & 0.16 & 0.20 & $2.8 \cdot 10^{3}$ \\
\hline 4 & 10 & 19 & 2.7 & 0.0038 & 0.0038 & 0.010 & $2.8 \cdot 10^{3}$ \\
\hline 5 & 17 & $1 \cdot 10^{-6}$ & 260 & 0.23 & 0.25 & 62 & $2.0 \cdot 10^{3}$ \\
\hline 6 & 17 & $2.7 \cdot 10^{-4}$ & 66 & 0.17 & 0.19 & 11 & $4.1 \cdot 10^{3}$ \\
\hline 7 & 17 & $7.1 \cdot 10^{-2}$ & 16 & 0.063 & 0.063 & 1.03 & $4.6 \cdot 10^{3}$ \\
\hline 8 & 17 & 19 & 4.0 & 0.016 & 0.015 & 0.066 & $5.1 \cdot 10^{3}$ \\
\hline 9 & 29 & $1 \cdot 10^{-6}$ & 390 & 0.30 & 0.31 & 120 & $2.1 \cdot 10^{3}$ \\
\hline 10 & 29 & $2.7 \cdot 10^{-4}$ & 98 & 0.25 & 0.26 & 24 & $5.7 \cdot 10^{3}$ \\
\hline 11 & 29 & $7.1 \cdot 10^{-2}$ & 24 & 0.13 & 0.13 & 3.2 & $8.8 \cdot 10^{3}$ \\
\hline 12 & 31 & 19 & 6.3 & 0.042 & 0.043 & 0.26 & $8.7 \cdot 10^{3}$ \\
\hline 13 & 29 & $5 \cdot 10^{3}$ & 1.5 & 0.0095 & 0.0095 & 0.014 & $8.6 \cdot 10^{3}$ \\
\hline 14 & 84 & $1 \cdot 10^{-6}$ & 870 & 0.34 & 0.34 & 290 & $1.3 \cdot 10^{3}$ \\
\hline 15 & 84 & $2.7 \cdot 10^{-4}$ & 220 & 0.30 & 0.32 & 64 & $4.7 \cdot 10^{3}$ \\
\hline 16 & 84 & $7.1 \cdot 10^{-2}$ & 54 & 0.25 & 0.25 & 14 & $1.2 \cdot 10^{4}$ \\
\hline 17 & 84 & 19 & 13 & 0.11 & 0.11 & 1.5 & $1.5 \cdot 10^{4}$ \\
\hline 18 & 84 & $5 \cdot 10^{3}$ & 3.3 & 0.030 & 0.031 & 0.099 & $1.4 \cdot 10^{4}$ \\
\hline 19 & 240 & $1 \cdot 10^{-6}$ & 1900 & 0.36 & 0.34 & 700 & $2.8 \cdot 10^{2}$ \\
\hline 20 & 240 & $2.7 \cdot 10^{-4}$ & 480 & 0.34 & 0.34 & 160 & $2.5 \cdot 10^{3}$ \\
\hline 21 & 240 & $7.1 \cdot 10^{-2}$ & 120 & 0.31 & 0.31 & 37 & $8.3 \cdot 10^{3}$ \\
\hline 22 & 240 & 19 & 30 & 0.21 & 0.20 & 3.2 & $1.8 \cdot 10^{4}$ \\
\hline 23 & 240 & $5 \cdot 10^{3}$ & 7.3 & 0.071 & 0.070 & 0.52 & $1.8 \cdot 10^{4}$ \\
\hline 24 & 700 & 19 & 65 & 0.28 & 0.28 & 18 & $1.3 \cdot 10^{4}$ \\
\hline 25 & 700 & $5 \cdot 10^{3}$ & 16 & 0.15 & 0.14 & 2.4 & $1.8 \cdot 10^{4}$ \\
\hline
\end{tabular}




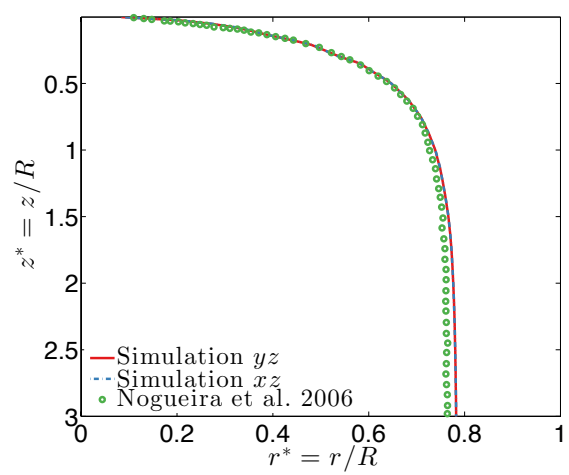

Figure B.11: The Taylor bubble shape of case with $E o=192$ and $M o=4.75 \cdot 10^{-2}$ obtained numerically is compared with the experimental results from [50.

\section{AppendixB. Code validation}

In this section, tThe bubble shape calculated by the CFD code is compared with experimental data from [50] for a case with $E o=192$ and $M o=$ $4.75 \cdot 10^{-2}$ (figure B.11), and in-house experiments generated with the experimental setup described later in AppendixC for $E o=57$ and $M o=3.1 \cdot 10^{-9}$

(figures B.12). Moreover, and-the calculated velocity vectors are compared to experimental PIV data from [14] for a case with $E o=98.4$ and $M o=$ $1.52 \cdot 10^{-2}$ (figures B.13 to B.15). Further validation results of the numerical method used in this study can be found in [40, 42, 41, e.g. the average error of the numerically obtained Taylor bubble velocity with respect to inclined in-house experiments is $-1.4 \% \pm 3.3 \%$.

In figure B.11, the bubble profile in the $y z$-plane (where the $z$-axis is the vertical) overlaps with the bubble profile in the perpendicular $x z$-plane, confirming an axisymmetric profile. On the other hand, figure B.12 depicts the tip bubble shape comparison for two inclined pipes $\left(\theta=30^{\circ}\right.$ and $\left.5^{\circ}\right)$ with $25 \%$ DI water and $75 \%$ methanol in volume $\left(E o=57, M o=3.1 \cdot 10^{-9}\right)$, ensuring that the numerical method employed can be applied to Taylor bubbles in inclined pipes.

Figure B.13a shows the axial velocity component along the tube axis above 


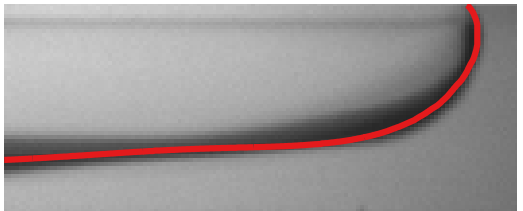

(a) $\theta=30^{\circ}$

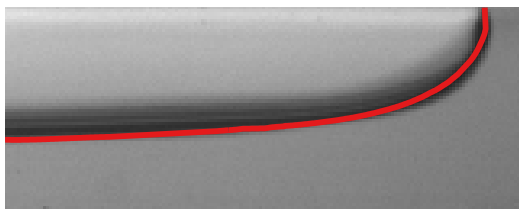

(b)

Figure B.12: Tip bubble shape comparison between experimental and numerical results for the mixture of $25 \%$ DI water and $75 \%$ methanol in volume and two different inclination angles.

the tip of the bubble: note that the presence of the bubble does not affect the flow beyond one diameter ahead of its tip. The axial and radial velocity components at $z / R=0.222$ above the bubble tip are shown on figure B.13 $\mathrm{b}$. The axial velocity is positive in the center region of the pipe, and becomes negative in the periphery due to the suction of the film around the Taylor bubble. The radial velocity is zero at the tube axis due to symmetry and at $z / R=1$ due to the tube wall, and is positive elsewhere since liquid is moving from the center region towards the pipe wall where it is suctioned by the liquid film. Figure B.14a shows the axial and radial velocity components in the film transition region at $z / R=1.008$ below the bubble nose. The film is still developing since the radial velocity is not zero there. The axial velocity is negative in the whole film region. PIV particles are only observed in the liquid region, and the absence of them indicates the presence of the gas phase. Figure B.14b shows the axial velocity profile in the fully developed falling film at a distance $z / R=4.64$ from the bubble nose. In this fully developed region, the radial velocity is zero as expected. Finally, figure B.15 depicts the axial and radial velocity components in the wake of the bubble at a distance $z / R=0.4$ below the bubble tail. The radial velocity is negative since the liquid coming from the falling film at the pipe wall moves towards the pipe inner core. Furthermore, the axial velocity is positive in the inner core, and negative in the outer core due to the falling film, a characteristic of the recirculation taking place in this case's bubble wake. The comparison between simulations and experiments is successful and provides confidence in the use of the commercial code in this study.

\footnotetext{
${ }^{2}$ Note that error bars were not provided in their article
} 

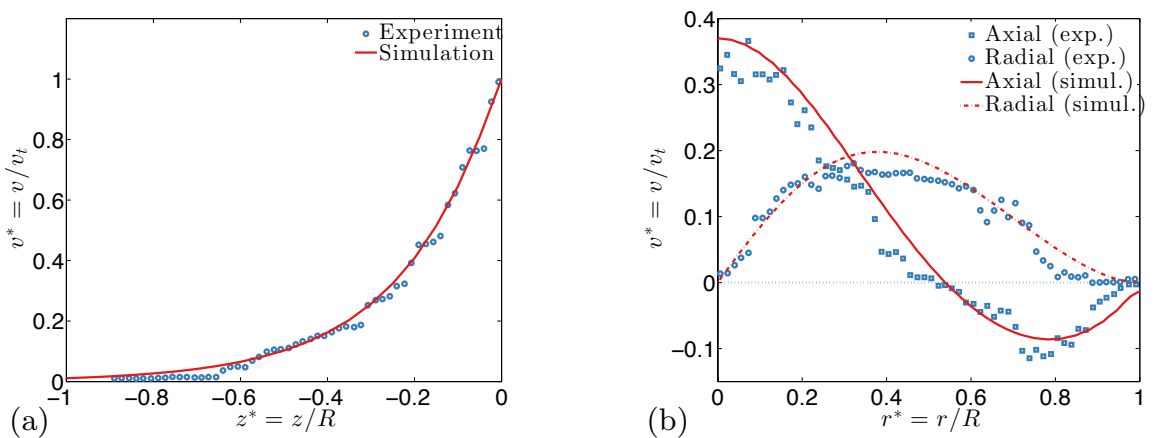

Figure B.13: The velocity profile of case with $E_{o}=98.4$ and $M o=1.52 \cdot 10^{-2}$ ahead of the bubble in the pipe centerline (a) and across the radial axis (b) obtained numerically is compared with the experimental data of $[14]^{2}$
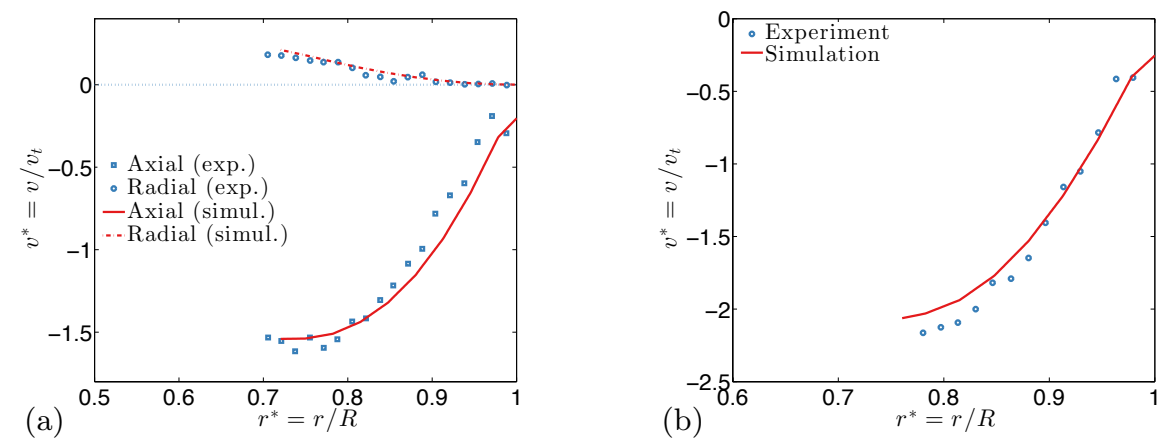

Figure B.14: The velocity profile of case with $E o=98.4$ and $M o=1.52 \cdot 10^{-2}$ in the developing region of the falling film (a) and in the developed film (b) obtained numerically is compared with the experimental data of [14]. 


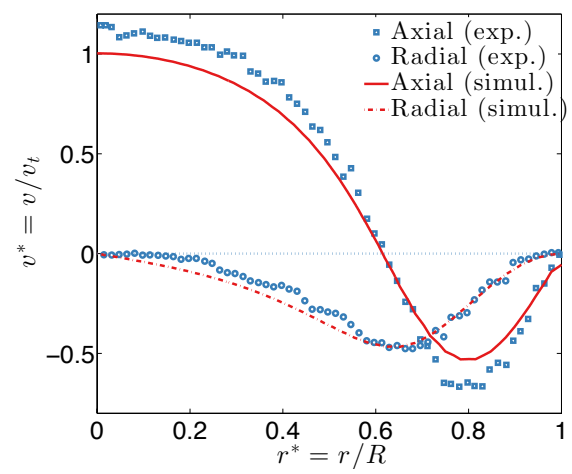

Figure B.15: The velocity profile of case with $E o=98.4$ and $M o=1.52 \cdot 10^{-2}$ in the wake of the bubble along the radial axis obtained numerically is compared with the experimental data of [14].

\section{AppendixC. Effect of Taylor bubble length on its terminal velocity and shape in inclined pipes}

It is well known that the dynamics of Taylor bubbles in vertical pipes are independent of their length [27, 44, 45, 69. However, the effect of bubble length in inclined pipes has only been mentioned by [77, who stated that, in his experiments, "the propagation velocity is independent of length as long as the volume of the bubble corresponds to a cylinder with the tube radius and a length of three tube radii". Here, we present both experiments and simulations showing that not only the terminal velocity but also the Taylor bubble shape are independent of its length in inclined pipes.

We measure the terminal velocity of Taylor bubbles with different volumes in inclined pipes. We analyze four liquids (methanol, ethanol, DI water, and a mixture of $50 \%$ DI water and $50 \%$ methanol in volume) at five inclination angles $\left(\theta=5^{\circ}, 15^{\circ}, 30^{\circ}, 37.5^{\circ}, 45^{\circ}\right)$. The experimental setup used [43], shown in figure C.16. consists of a High Speed Camera (HSC), a compressor, a polycarbonate tube of diameter $d=0.0127 \mathrm{~m}$, and three valves. The experimental procedure is as following: first, air at atmospheric pressure is introduced and held between the closed valves at "b" and "c". Then, valve "c" is opened and the Taylor 


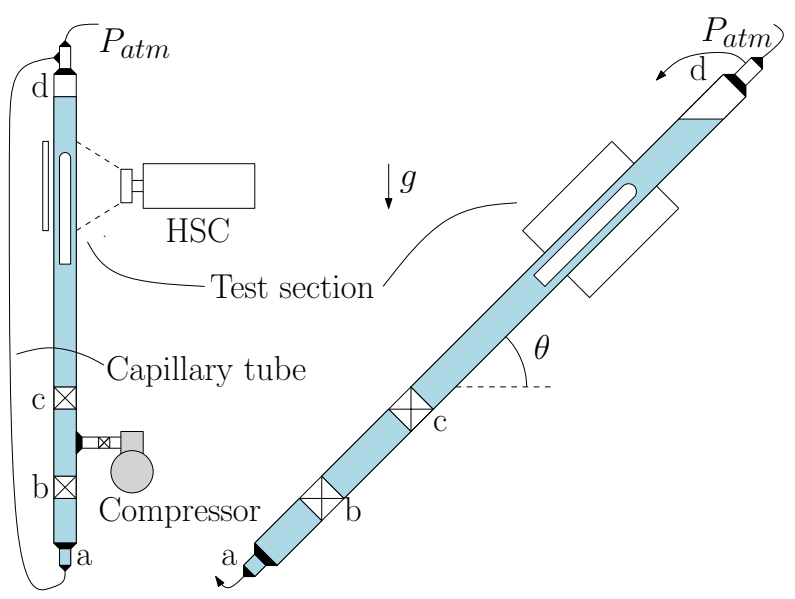

Figure C.16: Experimental setup (not drawn to scale)

bubble advances up through the tube due to buoyancy. The HSC records the bubble movement once it has reached its terminal velocity. The experiment finishes when the bubble reaches point "d".

For each liquid and inclination angle, several measurements are taken. In the first five measurements, $i=1, \ldots, 5$, valve "c" is maintained open and the compartment "b"- "c" fills up completely with liquid. In the following measurements, $i>5$, valve "c" is closed progressively earlier in time so that air remains in the compartment "b"- "c" and the volume of the experimental bubble rising the tube is progressively smaller, $V_{i} \geq V_{i+1}$. For all four fluids and five inclination angles, the measured terminal velocity was found to vary by no more than $1 \%$ when the bubble volume was varied up to $85 \%$ with respect to $V_{1}$, corresponding to approximately the same bubble length variation. This indicates that the bubble volume and length do not affect significantly the terminal velocity.

Numerical simulations also confirm that the terminal velocity and bubble shape do not depend on the bubble volume/length. Here we analyze a fluid with $M o=19$ and $E o=31$ at two inclination angles $\left(\theta=60^{\circ}, 45^{\circ}\right)$. Bubble volumes are characterized by $\lambda=1.05,1.17$, where the parameter $\lambda$ is calculated as $\lambda=d_{e} / d$, where $d_{e}$ is the sphere-volume equivalent diameter 
of the bubble. The differences between the terminal velocities of both volumes are $0.07 \%$ and $0.2 \%$ for $\theta=60^{\circ}$ and $\theta=45^{\circ}$, respectively. Furthermore, figure C.17 shows that the bubble tip shapes overlap for both inclination angles.
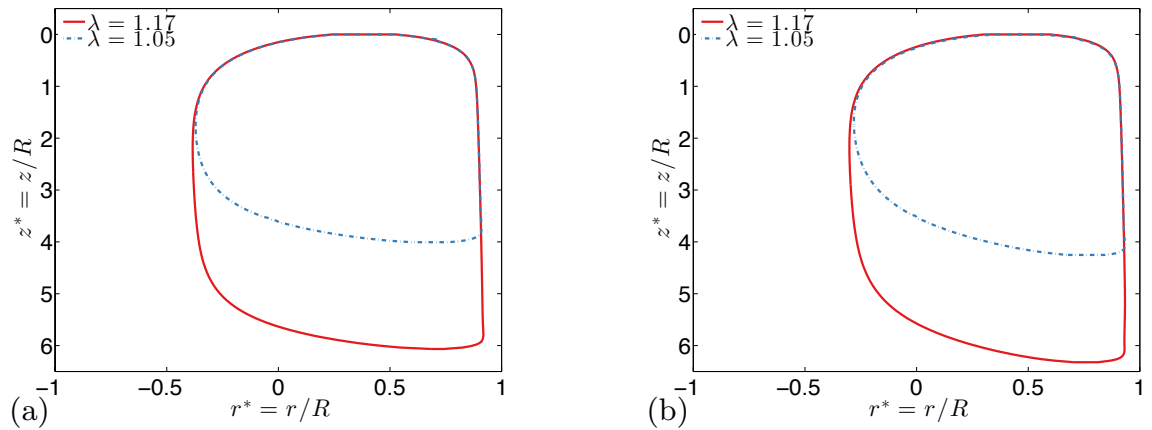

Figure C.17: Taylor bubble shapes in a liquid with $M o=19$ and $E o=31$ overlap for two different bubble volumes $(\lambda=1.17,1.05)$ for $\theta=60^{\circ}$ (a) and $\theta=45^{\circ}$

Thus, it is concluded that the Taylor bubble length does not affect its dynamics in inclined pipes, which is an important assumption made in the analysis described in section 3.2

\section{AppendixD. Statistical parameters}

$E_{1}$ is the average relative error in percentage points,

$$
E_{1}=\left(\frac{1}{N} \sum_{i}^{N} \frac{F r_{p r e d, i}-F r_{\text {sim }, i}}{F r_{\text {sim }, i}}\right) \cdot 100,
$$

where $N$ is the number of points, $F r_{p r e d, i}$ is the predicted $F r$ value of case $i$ using a correlation, and $F r_{s i m, i}$ is the numerically obtained $F r$ value of case $i$. $E_{2}$ is the absolute average relative error in percentage points,

$$
E_{2}=\left(\frac{1}{N} \sum_{i}^{N} \frac{\left|F r_{p r e d, i}-F r_{s i m, i}\right|}{F r_{s i m, i}}\right) \cdot 100
$$

$E_{3}$ is the standard deviation of the relative error in percentage points,

$$
E_{3}=\sqrt{\frac{1}{N-1} \sum_{i}^{N}\left(\frac{F r_{p r e d, i}-F r_{s i m, i}}{F r_{s i m, i}}-\frac{E_{1}}{100}\right)^{2}} \cdot 100
$$


$E_{4}$ is the average error in basis points,

$$
E_{4}=\frac{1}{N} \sum_{i}^{N}\left(F r_{\text {pred }, i}-F r_{\text {sim }, i}\right),
$$

$E_{5}$ is the absolute average error in basis points,

$$
E_{5}=\frac{1}{N} \sum_{i}^{N}\left|F r_{p r e d, i}-F r_{s i m, i}\right|,
$$

$E_{6}$ is the standard deviation of the absolute error in basis points,

$$
E_{6}=\sqrt{\frac{1}{N-1} \sum_{i}^{N}\left(F r_{p r e d, i}-F r_{s i m, i}-E_{4}\right)^{2}},
$$

and finally, $R^{2}$ is the coefficient of determination, a number that indicates how well data fit a model,

$$
R^{2}=1-\frac{S S_{r e s}}{S S_{t o t}}
$$

where $S S_{\text {res }}$ is the sum of squares of the residuals, also called the residual sum of squares, and $S S_{t o t}$ is the total sum of squares which is proportional to the variance of the data:

$$
\begin{aligned}
S S_{\text {res }} & =\sum_{i}^{N}\left(F r_{\text {sim }, i}-F r_{\text {pred }, i}\right)^{2} \\
S S_{\text {tot }} & =\sum_{i}^{N}\left(F r_{\text {sim }, i}-\bar{F} r_{\text {sim }}\right)^{2}
\end{aligned}
$$

${ }_{445}$ where $\bar{F} r_{\text {sim }}$ is the mean of the observed data, $\bar{F} r_{\text {sim }}=\sum_{i}^{N} F r_{\text {sim }, i} / N$.

\section{References}

[1] Alves, I. N., Shoham, O., \& Taitel, Y. (1993). Drift velocity of elongated bubbles in inclined pipes. Chemical Engineering Science, 48, 3063-3070.

[2] Ansari, A. M., Sylvester, N. D., Sarica, C., Shoham, O., \& Brill, J. P. (1994). A 
[3] Araújo, J. D. P., Miranda, J. M., \& Campos, J. B. L. M. (2013). Flow of two consecutive Taylor bubbles through a vertical column of stagnant liquid-A CFD study about the influence of the leading bubble on the hydrodynamics of the trailing one. Chemical Engineering Science, 97, 16-33.

[4] Araújo, J. D. P., Miranda, J. M., Pinto, A. M. F. R., \& Campos, J. B. L. M. (2012). Wide-ranging survey on the laminar flow of individual Taylor bubbles rising through stagnant Newtonian liquids. International Journal of Multiphase Flow, 43, 131-148.

[5] Balay, S., Gropp, W. D., McInnes, L. C., \& Smith, B. F. (1997). Efficient management of parallelism in object-oriented numerical software libraries. In Modern Software Tools for Scientific Computing (pp. 163-202). Springer.

[6] Beckermann, C., Diepers, H.-J., Steinbach, I., Karma, A., \& Tong, X. (1999). Modeling melt convection in phase-field simulations of solidification. Journal of Computational Physics, 154, 468-496.

[7] Bendiksen, K. H. (1984). An experimental investigation of the motion of long bubbles in inclined tubes. International Journal of Multiphase Flow, 10, 467483.

[8] Bendiksen, K. H., Malnes, D., \& Nydal, O. J. (1996). On the modelling of slug flow. Chemical Engineering Communications, 141, 71-102.

[9] Benjamin, T. B. (1968). Gravity currents and related phenomena. Journal of Fluid Mechanics, 31, 209-248.

[10] Bonnecaze, R. H., Erskine, W., \& Greskovich, E. J. (1971). Holdup and pressure drop for two-phase slug flow in inclined pipelines. AIChE Journal, 17, 1109-1113.

[11] Brill, J. P., \& Mukherjee, H. (1999). Multiphase flow in wells. Monograph Vol. 17, SPE Henry L. Dogorty Series, .

[12] British Petroleum (2011). Heavy Oil vs. Light Oil. Technical Report BP. URL: http://www.aoga.org/wp-content/uploads/2011/03/HRES-3.10. 11-Lunch-Learn-BP-Heavy-0il1.pdf 
[13] Bugg, J. D., Mack, K., \& Rezkallah, K. S. (1998). A numerical model of Taylor bubbles rising through stagnant liquids in vertical tubes. International Journal of Multiphase Flow, 24, 271-281.

[14] Bugg, J. D., \& Saad, G. A. (2002). The velocity field around a Taylor bubble rising in a stagnant viscous fluid: numerical and experimental results. International Journal of Multiphase Flow, 28, 791-803.

[15] Carew, P. S., Thomas, N. H., \& Johnson, A. B. (1995). A physically based correlation for the effects of power law rheology and inclination on slug bubble rise velocity. International Journal of Multiphase Flow, 21, 1091-1106. Printed.

[16] Cheng, H., Hills, J. H., \& Azzorpardi, B. J. (1998). A study of the bubble-to-slug transition in vertical gas-liquid flow in columns of different diameter. International Journal of Multiphase Flow, 24, 431-452.

[17] Chung, M.-H. (2001). A level set approach for computing solutions to inviscid compressible flow with moving solid boundary using fixed cartesian grids. International Journal for Numerical Methods in Fluids, 36, 373-389.

[18] Clarke, A., \& Issa, R. I. (1997). A numerical model of slug flow in vertical tubes. Computers \& Fluids, 26, 395-415.

[19] Clift, R., Grace, J. R., \& Weber, M. E. (2005). Bubbles, Drops, and Particles. Dover Publications, Inc.

[20] Davies, R. M., \& Taylor, G. (1950). The mechanics of large bubbles rising through extended liquids and through liquids in tubes. Proceedings of the Royal Society of London. Series A. Mathematical and Physical Sciences, 200, 375-390.

[21] Doormaal, J. P. V., \& Raithby, G. D. (1984). Enhancements of the SIMPLE method for predicting incompressible fluid flows. Numerical Heat Transfer, 7, $147-163$.

[22] Dukler, A. E., \& Hubbard, M. G. (1975). A model for gas-liquid slug flow in horizontal and near horizontal tubes. Industrial \& Engineering Chemistry Fundamentals, 14, 337-347. 
[23] Dumitrescu, D. T. (1943). Strömung an einer Luftblase im senkrechten Rohr. ZAMM-Journal of Applied Mathematics and Mechanics/Zeitschrift für Angewandte Mathematik und Mechanik, 23, 139-149.

[24] Fabre, J., \& Liné, A. (1992). Modeling of two-phase slug flow. Annual Review of Fluid Mechanics, 24, 21-46.

[25] Falgout, R. D., Jones, J. E., \& Yang, U. M. (2006). The design and implementation of hypre, a library of parallel high performance preconditioners. In Numerical Solution of Partial Differential Equations on Parallel Computers (pp. 267-294). Springer.

[26] Gokcal, B., Al-Sarkhi, A. S., \& Sarica, C. (2009). Effects of high oil viscosity on drift velocity for horizontal and upward inclined pipes. SPE Projects Facilities \& Construction, 4, 32-40.

520 [27] Griffith, P., \& Wallis, G. B. (1961). Two-phase slug flow. Journal of Heat Transfer, 83, 307-318.

[28] Hasan, A. R., \& Kabir, C. S. (1988). Predicting multiphase flow behavior in a deviated well. SPE Production Engineering, 3, 474-482.

[29] Hayashi, K., Kurimoto, R., \& Tomiyama, A. (2011). Terminal velocity of a Taylor drop in a vertical pipe. International Journal of Multiphase Flow, 37, 241-251.

[30] Jepson, W. P., \& Taylor, R. E. (1993). Slug flow and its transitions in largediameter horizontal pipes. International Journal of Multiphase Flow, 19, 411420.

[31] Jeyachandra, B. C., Gokcal, B., Al-Sarkhi, A., Sarica, C., \& Sharma, A. (2012). Drift-velocity closure relationships for slug two-phase high-viscosity oil flow in pipes. SPE Journal, 17, 593-601. Printed.

[32] Joseph, D. D. (2003). Rise velocity of a spherical cap bubble. Journal of Fluid Mechanics, 488, 213-223.

[33] Joseph, D. D., \& Yang, B. H. (2010). Friction factor correlations for laminar, 535 transition and turbulent flow in smooth pipes. Physica D: Nonlinear Phenomena, 239, 1318-1328. 
[34] Kang, C.-W., Quan, S., \& Lou, J. (2010). Numerical study of a Taylor bubble rising in stagnant liquids. Phys. Rev. E, 81, 066308. URL: http://link.aps. org/doi/10.1103/PhysRevE.81.066308, doi 10.1103/PhysRevE.81.066308

[35] Kataoka, I., \& Ishii, M. (1987). Drift-flux model for large diameter pipe and new correlation for pool void fraction. International Journal of Heat and Mass Transfer, 30, 1927-1939.

[36] Kocamustafaogullari, G., \& Ishii, M. (1985). Maximum fluid particle size for bubbles and drops. In Fundamental Aspects of Gas-Liquid Flows.

[37] Kurimoto, R., Hayashi, K., \& Tomiyama, A. (2013). Terminal velocities of clean and fully-contaminated drops in vertical pipes. International Journal of Multiphase Flow, 49, 8-23.

[38] Labois, M., Panyasantisuk, J., Höhne, T., Kliem, S., \& Lakehal, D. (2010). On the prediction of boron dilution using the CMFD code TRANSAT: the ROCOM test case. In Proc. CFD4NRS 4. OECD-NEA, Washington, USA.

[39] Lakehal, D., Meier, M., \& Fulgosi, M. (2002). Interface tracking towards the direct simulation of heat and mass transfer in multiphase flows. International Journal of Heat and Fluid Flow, 23, 242 - 257. URL: http: //www.sciencedirect.com/science/article/pii/S0142727X02001728 doi:10. 1016/S0142-727X(02)00172-8.

[40] Lizarraga-Garcia, E. (2016). A study of Taylor bubbles in vertical and inclined slug flow using multiphase CFD with level set. Ph.D. thesis Massachusetts Institute of Technology.

[41] Lizarraga-Garcia, E., Buongiorno, J., Al-Safran, E., \& Lakehal, D. (2015). CFDinformed unified closure relation for the rise velocity of Taylor bubbles in pipes. Bulletin of the American Physical Society, 60.

[42] Lizarraga-Garcia, E., Buongiorno, J., Al-Safran, E., \& Lakehal, D. (2015). Development of a new CFD-based unified closure relation for Taylor bubble velocity in two-phase slug flow in pipes. In 17th International Conference on Multiphase Production Technology, Cannes, France (pp. 93-107). BHR Group. 
[43] Lizarraga-Garcia, E., Buongiorno, J., \& Bucci, M. (2016). An analytical film drainage model and breakup criterion for Taylor bubbles in slug flow in inclined round pipes. International Journal of Multiphase Flow, 84, 46-53.

[44] Llewellin, E. W., Bello, E. D., Taddeucci, J., Scarlato, P., \& Lane, S. J. (2012). The thickness of the falling film of liquid around a Taylor bubble. Proceedings of the Royal Society A: Mathematical, Physical and Engineering Science, 468, 1041-1064.

[45] Mao, Z.-S., \& Dukler, A. (1989). An experimental study of gas-liquid slug flow. Experiments in Fluids, 8, 169-182.

575 [46] Mao, Z.-S., \& Dukler, A. E. (1990). The motion of Taylor bubbles in vertical tubes-I. A numerical simulation for the shape and rise velocity of Taylor bubbles in stagnant and flowing liquid. Journal of Computational Physics, 91, 132 - 160. URL: http://www.sciencedirect.com/science/article/pii/ 0021999190900080, doi 10.1016/0021-9991(90)90008-0.

[47] MATLAB $^{\circledR}$ (2013). version 8.2.0.701 (R2013b). The MathWorks Inc., Natick, Massachusetts, .

[48] Moreiras, J., Pereyra, E., Sarica, C., \& Torres, C. F. (2014). Unified drift velocity closure relationship for large bubbles rising in stagnant viscous fluids in pipes. Journal of Petroleum Science and Engineering, 124, 359-366.

[49] Nicklin, D. J., Wilkes, J. O., \& Davidson, J. F. (1962). Two-phase flow in vertical tubes. Trans. Inst. Chem. Eng, 40, 61-68.

[50] Nogueira, S., Riethmuler, M. L., Campos, J. B. L. M., \& Pinto, A. M. F. R. (2006). Flow in the nose region and annular film around a Taylor bubble rising through vertical columns of stagnant and flowing Newtonian liquids. Chemical Engineering Science, 61, 845 - 857. doi http://dx.doi.org/10.1016/j.ces.2005.07.038

[51] Oddie, G., Shi, H., Durlofsky, L. J., Aziz, K., Pfeffer, B., \& Holmes, J. A. (2003). Experimental study of two and three phase flows in large diameter inclined pipes. International Journal of Multiphase Flow, 29, 527-558. 
[52] Ohnuki, A., \& Akimoto, H. (2000). Experimental study on transition of flow pattern and phase distribution in upward air-water two-phase flow along a large vertical pipe. International Journal of Multiphase Flow, 26, 367-386.

[53] Orell, A., \& Rembrand, R. (1986). A model for gas-liquid slug flow in a vertical tube. Industrial \& Engineering Chemistry Fundamentals, 25, 196-206.

[54] Osher, S., \& Sethian, J. A. (1988). Fronts propagating with curvature-dependent speed: algorithms based on Hamilton-Jacobi formulations. Journal of Computational Physics, 79, 12-49.

[55] Patankar, N. A., Joseph, D. D., Wang, J., Barree, R. D., Conway, M., \& Asadi, M. (2002). Power law correlations for sediment transport in pressure driven channel flows. International Journal of Multiphase Flow, 28, 1269-1292.

[56] Peng, D. J., Ahmad, A., Hale, C. P., Matar, O. K., \& Hewitt, G. F. (2010). Flow regime transition in large diameter pipes. In 7th International Conference on Multiphase Flow (ICMF 2010), Tampa, FL USA.

[57] Petalas, N., \& Aziz, K. (2000). A mechanistic model for multiphase flow in pipes. Journal of Canadian Petroleum Technology, 39, 43-55.

[58] Prasser, H.-M., Beyer, M., Böttger, A., Carl, H., Lucas, D., Schaffrath, A., Schütz, P., Weiss, F.-P., \& Zschau, J. (2005). Influence of the pipe diameter on the structure of the gas-liquid interface in a vertical two-phase pipe flow. Nuclear Technology, 152, 3-22.

[59] R-cycle (2016). Personal communication, .

615 [60] Ramdin, M., \& Henkes, R. (2012). Computational fluid dynamics modeling of Benjamin and Taylor bubbles in two-phase flow in pipes. Journal of Fluids Engineering, 134, 041303.

[61] Saad, Y., \& Schultz, M. H. (1986). GMRES: A generalized minimal residual algorithm for solving nonsymmetric linear systems. SIAM Journal on Scientific and Statistical Computing, 7, 856-869.

[62] Shosho, C. E., \& Ryan, M. E. (2001). An experimental study of the motion of long bubbles in inclined tubes. Chemical Engineering Science, 56, 2191-2204. 
[63] Spedding, P. L., \& Nguyen, V. T. (1978). Bubble rise and liquid content in horizontal and inclined tubes. Chemical Engineering Science, 33, 987-994.

625 [64] Stanislav, J. F., Kokal, S., \& Nicholson, M. K. (1986). Intermittent gas-liquid flow in upward inclined pipes. International Journal of Multiphase Flow, 12, 325-335.

[65] Stone, H. L. (1968). Iterative solution of implicit approximations of multidimensional partial differential equations. SIAM Journal on Numerical Analysis, 5, $530-558$.

${ }_{630}^{606]}$ Sussman, M., Smereka, P., \& Osher, S. (1994). A level set approach for computing solutions to incompressible two-phase flow. Journal of Computational Physics, $114,146-159$.

[67] Taha, T., \& Cui, Z. F. (2006). CFD modelling of slug flow in vertical tubes. Chemical Engineering Science, 61, 676-687.

[68] Taitel, Y., \& Barnea, D. (1990). Two-phase slug flow. Advances in Heat Transfer, 20, 83-132.

[69] Tomiyama, A., Nakahara, Y., \& Morita, G. (2001). Rising velocities and shapes of single bubbles in vertical pipes. International Conference of Multiphase Flow, (pp. 1-12).

[70] TransAT ${ }^{\circledR}$ (2014). version 5.1.1. ASCOMP GmbH, Zurich, Switzerland, .

[71] Viana, F., Pardo, R., Yanez, R., Trallero, J. L., \& Joseph, D. D. (2003). Universal correlation for the rise velocity of long gas bubbles in round pipes. Journal of Fluid Mechanics, 494, 379-398.

[72] Vrij, A. (1966). Possible mechanism for the spontaneous rupture of thin, free liquid films. Discussions of the Faraday Society, 42, 23-33.

[73] Wallis, G. B. (1969). One-Dimensional Two-Phase Flow volume 1. McGraw-Hill New York.

[74] Weber, M. E., Alarie, A., \& Ryan, M. E. (1986). Velocities of extended bubbles in inclined tubes. Chemical Engineering Science, 41, 2235-2240. Printed. 
[75] White, E. T., \& Beardmore, R. H. (1962). The velocity of rise of single cylindrical air bubbles through liquids contained in vertical tubes. Chemical Engineering Science, 17, 351-361.

[76] Zhu, J. (1991). A low-diffusive and oscillation-free convection scheme. Communications in Applied Numerical Methods, 7, 225-232.

655 [77] Zukoski, E. E. (1966). Influence of viscosity, surface tension, and inclination angle on motion of long bubbles in closed tubes. Journal of Fluid Mechanics, 25, 821-837. 